\title{
Determinant Representations of Polynomial Sequences of Riordan Type
}

\author{
Sheng-liang Yang and Sai-nan Zheng \\ Department of Applied Mathematics, Lanzhou University of Technology, Lanzhou 730050, China \\ Correspondence should be addressed to Sheng-liang Yang; slyang@lut.cn
}

Received 12 November 2012; Revised 19 January 2013; Accepted 27 January 2013

Academic Editor: Gi Sang Cheon

Copyright ( 2013 S.-l. Yang and S.-n. Zheng. This is an open access article distributed under the Creative Commons Attribution License, which permits unrestricted use, distribution, and reproduction in any medium, provided the original work is properly cited.

In this paper, using the production matrix of a Riordan array, we obtain a recurrence relation for polynomial sequence associated with the Riordan array, and we also show that the general term for the sequence can be expressed as the characteristic polynomial of the principal submatrix of the production matrix. As applications, a unified determinant expression for the four kinds of Chebyshev polynomials is given.

\section{Introduction}

The concept of a Riordan array is very useful in combinatorics. The infinite triangles of Pascal, Catalan, Motzkin, and Schröder are important and meaningful examples of Riordan array, and many others have been proposed and developed (see, e.g., [1-7]). In the recent literature, Riordan arrays have attracted the attention of various authors from many points of view and many examples and generalizations can be found (see, e.g., [8-12]).

A Riordan array denoted by $(g(t), f(t))$ is an infinite lower triangular matrix such that its column $k(k=$ $0,1,2, \ldots)$ has generating function $g(t) f(t)^{k}$, where $g(t)=$ $\sum_{n=0}^{\infty} g_{n} t^{n}$ and $f(t)=\sum_{n=0}^{\infty} f_{n} t^{n}$ are formal power series with $g_{0}=1, f_{0}=0$, and $f_{1} \neq 0$. That is, the general term of matrix $R=(g(t), f(t))$ is $r_{n, k}=\left[t^{n}\right] g(t) f(t)^{k}$; here $\left[t^{n}\right] h(t)$ denotes the coefficient of $t^{n}$ in power series $h(t)$. Given a Riordan array $(g(t), f(t))$ and column vector $B=\left(b_{0}, b_{1}, b_{2}, \ldots\right)^{T}$, the product of $(g(t), f(t))$ and $B$ gives a column vector whose generating function is $g(t) b(f(t))$, where $b(t)=\sum_{n=0}^{\infty} b_{n} t^{n}$. If we identify a vector with its ordinary generating function, the composition rule can be rewritten as

$$
(g(t), f(t)) b(t)=g(t) b(f(t)) .
$$

This property is called the fundamental theorem for Riordan arrays and this leads to the matrix multiplication for Riordan arrays:

$$
(g(t), f(t))(h(t), l(t))=(g(t) h(f(t)), l(f(t))) .
$$

The set of all Riordan arrays forms a group under the previos operation of a matrix multiplication. The identity element of the group is $(1, t)$. The inverse element of $(g(t), f(t))$ is

$$
(g(t), f(t))^{-1}=\left(\frac{1}{g(\bar{f}(t))}, \bar{f}(t)\right),
$$

where $\bar{f}(t)$ is compositional inverse of $f(t)$.

A Riordan array $R=(g(t), f(t))=\left(r_{n, k}\right)_{n, k \geq 0}$ can be characterized by two sequences $A=\left(a_{i}\right)_{i \geq 0}$ and $Z=\left(z_{i}\right)_{i \geq 0}$ such that, for $n, k \geq 0$

$$
\begin{gathered}
r_{n+1,0}=z_{0} r_{n, 0}+z_{1} r_{n, 1}+z_{2} r_{n, 2}+\cdots, \\
r_{n+1, k+1}=a_{0} r_{n, k}+a_{1} r_{n, k+1}+a_{2} r_{n, k+2}+\cdots .
\end{gathered}
$$

If $A(t)$ and $Z(t)$ are the generating functions for the $A$ - and $Z$-sequences, respectively, then it follows that $[9,13]$

$$
g(t)=\frac{1}{1-t Z(f(t))}, \quad f(t)=t A(f(t)) .
$$


If the inverse of $R=(g(t), f(t))$ is $R^{-1}=(d(t), h(t))$, then the $A$ - and $Z$-sequences of $R$ are

$$
A(t)=\frac{t}{h(t)}, \quad Z(t)=\frac{1}{h(t)}(1-d(t)) .
$$

For an invertible lower triangular matrix $R$, its production matrix (also called its Stieltjes matrix; see $[11,14]$ ) is the matrix $P=R^{-1} \bar{R}$, where $\bar{R}$ is the matrix $R$ with its first row removed. The production matrix $P$ can be characterized by the matrix equality $R P=D R$, where $D=\left(\delta_{i+1, j}\right)_{i, j \geq 0}$ ( $\delta$ is the usual Kronecker delta).

Lemma 1 (see [14]). Assume that $R=\left(r_{n, k}\right)$ is an infinite lower triangular matrix with $r_{n, n} \neq 0$. Then $R$ is a Riordan array if and only if its production matrix $P$ is of the form

$$
P=\left(\begin{array}{ccccccc}
z_{0} & a_{0} & 0 & 0 & 0 & 0 & \cdots \\
z_{1} & a_{1} & a_{0} & 0 & 0 & 0 & \cdots \\
z_{2} & a_{2} & a_{1} & a_{0} & 0 & 0 & \cdots \\
z_{3} & a_{3} & a_{2} & a_{1} & a_{0} & 0 & \cdots \\
z_{4} & a_{4} & a_{3} & a_{2} & a_{1} & a_{0} & \cdots \\
\vdots & \vdots & \vdots & \vdots & \vdots & \vdots & \ddots
\end{array}\right)
$$

where $\left(a_{0}, a_{1}, a_{2}, \ldots\right)$ is the $A$-sequence and $\left(z_{0}, z_{1}, z_{2}, \ldots\right)$ is the $Z$-sequence of the Riordan array $R$.

Definition 2. Let $\left(r_{n}(x)\right)_{n \geq 0}$ be a sequence of polynomials where $r_{n}(x)$ is of degree $n$ and $r_{n}(x)=\sum_{k=0}^{n} r_{n, k} x^{k}$. We say that $\left(r_{n}(x)\right)_{n \geq 0}$ is a polynomial sequence of Riordan type if the coefficient matrix $\left(r_{n, k}\right)_{n, k \geq 0}$ is an element of the Riordan group; that is, there exists a Riordan array $(g(t), f(t))$ such that $\left(r_{n, k}\right)_{n, k \geq 0}=(g(t), f(t))$. In this case, we say that $\left(r_{n}(x)\right)_{n \geq 0}$ is the polynomial sequence associated with the Riordan array $(g(t), f(t))$.

Letting $r_{n}(x)=\sum_{k=0}^{n} r_{n, k} x^{k}, n \geq 0$, then in matrix form we have

$$
\left(\begin{array}{ccccc}
r_{0,0} & 0 & 0 & 0 & \cdots \\
r_{1,0} & r_{1,1} & 0 & 0 & \cdots \\
r_{2,0} & r_{2,1} & r_{2,2} & 0 & \cdots \\
r_{3,0} & r_{3,1} & r_{3,2} & r_{3,3} & \cdots \\
\vdots & \vdots & \vdots & \vdots & \ddots
\end{array}\right)\left(\begin{array}{c}
1 \\
x \\
x^{2} \\
x^{3} \\
\vdots
\end{array}\right)=\left(\begin{array}{c}
r_{0}(x) \\
r_{1}(x) \\
r_{2}(x) \\
r_{3}(x) \\
\vdots
\end{array}\right) .
$$

Hence, by using (1), we have the following lemma.

Lemma 3. Let $\left(r_{n}(x)\right)_{n \geq 0}$ be the polynomial sequence associated with a Riordan array $(g(t), f(t))$, and let $r(t, x)=$ $\sum_{n=0}^{\infty} r_{n}(x) t^{n}$ be its generating function. Then

$$
r(t, x)=\frac{g(t)}{1-x f(t)} .
$$

In [15], Luzón introduced a new notation $T(f \mid g)$ to represent the Riordan arrays and gave a recurrence relation for the family of polynomials associated to Riordan arrays. In recent works $[16,17]$, a new definition by means of a determinant form for Appell polynomials is given. Sequences of Appell polynomials are special of the Sheffer sequences [18]. In [19], the author obtains a determinant representation for the Sheffer sequence. The aim of this work is to propose a similar approach for polynomial sequences of Riordan type, which are special of the generalized Sheffer sequences [12, 18]. A determinant representation for polynomial sequences of Riordan type is obtained by using production matrix of Riordan array. In fact, we will show that the general formula for the polynomial sequences of Riordan type can be expressed as the characteristic polynomial of the principal submatrix of the production matrix. As applications, determinant expressions for some classical polynomial sequences such as Fibonacci, Pell, and Chebyshev are derived, and a unified determinant expression for the four kinds of Chebyshev polynomials $[20,21]$ is established.

\section{Main Theorem}

In this section we are going to develop our main theorem.

Theorem 4. Let $R=(g(t), f(t))$ be a Riordan array with the $Z$-sequence $\left(z_{i}\right)_{i \geq 0}$ and the A-sequence $\left(a_{i}\right)_{i \geq 0}$. Let $\left(p_{n}(x)\right)_{n \geq 0}$ be the polynomial sequence associated with $R^{-1}$. Then $\left(p_{n}(x)\right)_{n \geq 0}$ satisfies the recurrence relation:

$$
\begin{aligned}
a_{0} p_{n}(x)= & x p_{n-1}(x)-a_{1} p_{n-1}(x)-a_{2} p_{n-2}(x) \\
& -\cdots-a_{n-1} p_{1}(x)-z_{n-1} p_{0}(x), \quad n>1,
\end{aligned}
$$

with initial condition $p_{0}(x)=1$, and $a_{0} p_{1}(x)=x-z_{0}$. In general, for all $n \geq 1, p_{n}(x)$ is given by the following Hessenberg determinant:

$$
\begin{aligned}
& p_{n}(x) \\
& =(-1)^{n} a_{0}^{-n} \\
& \quad\left|\begin{array}{ccccccccc}
z_{0}-x & a_{0} & 0 & 0 & 0 & \cdots & 0 & 0 \\
z_{1} & a_{1}-x & a_{0} & 0 & 0 & \cdots & 0 & 0 \\
z_{2} & a_{2} & a_{1}-x & a_{0} & 0 & \cdots & 0 & 0 \\
z_{3} & a_{3} & a_{2} & a_{1}-x & a_{0} & \cdots & 0 & 0 \\
z_{4} & a_{4} & a_{3} & a_{2} & a_{1}-x & \cdots & 0 & 0 \\
\vdots & \vdots & \vdots & \vdots & \vdots & \ddots & \vdots & \vdots \\
z_{n-2} & a_{n-2} & a_{n-3} & a_{n-4} & a_{n-5} & \cdots & a_{1}-x & a_{0} \\
z_{n-1} & a_{n-1} & a_{n-2} & a_{n-3} & a_{n-4} & \cdots & a_{2} & a_{1}-x
\end{array}\right| .
\end{aligned}
$$

Proof. Let $R=(g(t), f(t))$ and $R^{-1}=(g(t), f(t))^{-1}=$ $(d(t), h(t))$. Then from definition and (3), we have $g(0)=1$ and $d(t)=1 / g(\bar{f}(t))$. Hence $d(0)=1$ and $p_{0}(x)=1$. Letting $E=\left(1, x, x^{2}, \ldots\right)^{T}$, then $R^{-1} E=\left(p_{0}(x), p_{1}(x), p_{2}(x), \ldots\right)^{T}$ and $D E=\left(x, x^{2}, x^{3}, \ldots\right)^{T}$, where $D=\left(\delta_{i+1, j}\right)$. Letting $P$ be the production matrix of $R$, then $R P=D R$, and $P R^{-1}=$ $R^{-1} D$. Thus $P R^{-1} E=R^{-1} D E$, and $P R^{-1} E=x R^{-1} E$. In matrix form, we have

$$
\left(\begin{array}{cccccc}
z_{0} & a_{0} & 0 & 0 & 0 & \cdots \\
z_{1} & a_{1} & a_{0} & 0 & 0 & \cdots \\
z_{2} & a_{2} & a_{1} & a_{0} & 0 & \cdots \\
z_{3} & a_{3} & a_{2} & a_{1} & a_{0} & \cdots \\
\vdots & \vdots & \vdots & \vdots & \vdots & \ddots
\end{array}\right)\left(\begin{array}{c}
p_{0}(x) \\
p_{1}(x) \\
p_{2}(x) \\
p_{3}(x) \\
\vdots
\end{array}\right)=\left(\begin{array}{c}
x p_{0}(x) \\
x p_{1}(x) \\
x p_{2}(x) \\
x p_{3}(x) \\
\vdots
\end{array}\right) .
$$


Using the block matrix method, we get

$$
\begin{gathered}
p_{0}(x)\left(\begin{array}{c}
z_{0} \\
z_{1} \\
z_{2} \\
z_{3} \\
\vdots
\end{array}\right)+\left(\begin{array}{ccccc}
a_{0} & 0 & 0 & 0 & \cdots \\
a_{1} & a_{0} & 0 & 0 & \cdots \\
a_{2} & a_{1} & a_{0} & 0 & \cdots \\
a_{3} & a_{2} & a_{1} & a_{0} & \cdots \\
\vdots & \vdots & \vdots & \vdots & \ddots
\end{array}\right) \\
\times\left(\begin{array}{c}
p_{1}(x) \\
p_{2}(x) \\
p_{3}(x) \\
p_{4}(x) \\
\vdots
\end{array}\right)=\left(\begin{array}{c}
x p_{0}(x) \\
x p_{1}(x) \\
x p_{2}(x) \\
x p_{3}(x) \\
\vdots
\end{array}\right) .
\end{gathered}
$$

Since

$$
\begin{aligned}
& \left(\begin{array}{c}
x p_{0}(x) \\
x p_{1}(x) \\
x p_{2}(x) \\
x p_{3}(x) \\
\vdots
\end{array}\right)=\left(\begin{array}{c}
x p_{0}(x) \\
0 \\
0 \\
0 \\
\vdots \\
0
\end{array}\right)+\left(\begin{array}{c}
0 \\
x p_{1}(x) \\
x p_{2}(x) \\
x p_{3}(x) \\
\vdots
\end{array}\right) \\
& \left(\begin{array}{c}
x p_{1}(x) \\
x p_{2}(x) \\
x p_{3}(x) \\
\vdots
\end{array}\right)=\left(\begin{array}{ccccc}
0 & 0 & 0 & 0 & \cdots \\
x & 0 & 0 & 0 & \cdots \\
0 & x & 0 & 0 & \cdots \\
0 & 0 & x & 0 & \cdots \\
\vdots & \vdots & \vdots & \vdots & \ddots
\end{array}\right)\left(\begin{array}{c}
p_{1}(x) \\
p_{2}(x) \\
p_{3}(x) \\
p_{4}(x) \\
\vdots
\end{array}\right) .
\end{aligned}
$$

The previous matrix equation can be rewritten as

$$
\begin{gathered}
\left(\begin{array}{ccccc}
a_{0} & 0 & 0 & 0 & \cdots \\
a_{1}-x & a_{0} & 0 & 0 & \cdots \\
a_{2} & a_{1}-x & a_{0} & 0 & \cdots \\
a_{3} & a_{2} & a_{1}-x & a_{0} & \cdots \\
\vdots & \vdots & \vdots & \vdots & \ddots
\end{array}\right)\left(\begin{array}{c}
p_{1}(x) \\
p_{2}(x) \\
p_{3}(x) \\
p_{4}(x) \\
\vdots
\end{array}\right) \\
=p_{0}(x)\left(\begin{array}{c}
x-z_{0} \\
-z_{1} \\
-z_{2} \\
-z_{3} \\
\vdots
\end{array}\right) .
\end{gathered}
$$

Therefore, $a_{0} p_{1}(x)=x-z_{0}$, and for $n>1$, we have

$$
\begin{aligned}
& a_{n-1} p_{1}(x)+\cdots+a_{2} p_{n-2}(x)+\left(a_{1}-x\right) p_{n-1}(x)+a_{0} p_{n}(x) \\
& \quad=-z_{n-1} p_{0}(x)
\end{aligned}
$$

or equivalently

$$
\begin{aligned}
a_{0} p_{n}(x)= & \left(x-a_{1}\right) p_{n-1}(x)-a_{2} p_{n-2}(x) \\
& -\cdots-a_{n-1} p_{1}(x)-z_{n-1} p_{0}(x) .
\end{aligned}
$$

By applying the Cramer's rule, we can work out the unknown $p_{n}(x)$ operating with the first $n$ equations in (15):

$$
p_{n}(x)=a_{0}^{-n}\left|\begin{array}{cccccccc}
a_{0} & 0 & 0 & 0 & 0 & \cdots & 0 & \left(x-z_{0}\right) p_{0}(x) \\
a_{1}-x & a_{0} & 0 & 0 & 0 & \cdots & 0 & -z_{1} p_{0}(x) \\
a_{2} & a_{1}-x & a_{0} & 0 & 0 & \cdots & 0 & -z_{2} p_{0}(x) \\
a_{3} & a_{2} & a_{1}-x & a_{0} & 0 & \cdots & 0 & -z_{3} p_{0}(x) \\
a_{4} & a_{3} & a_{2} & a_{1}-x & a_{0} & \cdots & 0 & -z_{4} p_{0}(x) \\
\vdots & \vdots & \vdots & \vdots & \vdots & \ddots & \vdots & \vdots \\
a_{n-2} & a_{n-3} & a_{n-4} & a_{n-5} & a_{n-6} & \cdots & a_{0} & -z_{n-2} p_{0}(x) \\
a_{n-1} & a_{n-2} & a_{n-3} & a_{n-4} & a_{n-5} & \cdots & a_{1}-x & -z_{n-1} p_{0}(x)
\end{array}\right|
$$

After transferring the last column to the first position, an operation which introduces the factor $(-1)^{n-1}$, the theorem follows.

Corollary 5. Let $R=(g(t), f(t))$ be a Riordan array with production matrix $P$. Let $\left(p_{n}(x)\right)_{n \geq 0}$ be the polynomial sequence associated with $R^{-1}=(g(t), f(t))^{-1}$. Then $p_{0}(x)=1$, and for all $n \geq 1$,

$$
p_{n}(x)=a_{0}^{-n} \operatorname{det}\left(x I_{n}-P_{n}\right),
$$

where $a_{0}=p_{0,1}, P_{n}$ is the principal submatrix of order $n$ of the production matrix $P$ and $I_{n}$ is the identity matrix of order $n$. 


\section{Applications}

A useful application of Theorem 4 is to find the determinant expression of a well-known sequence. We illustrate the ideal in the following examples. In the final paragraph, we will give a unified determinant expression for the four kinds of Chebyshev polynomials.

Example 6. Considering the Riordan array $\mathscr{A}=(1 /(1+$ $\left.r t^{2}\right)$, at $\left./\left(1+r t^{2}\right)\right)$, we have $\left(1 /\left(1+r t^{2}\right)\right.$, at $\left./\left(1+r t^{2}\right)\right)(1 /(1-$ $x t))=1 /\left(1-a x t+r t^{2}\right)$. The generating functions of the $A-$ and $Z$-sequences of $\mathscr{A}^{-1}$ are

$$
A(t)=\frac{1+r t^{2}}{a}, \quad Z(t)=\frac{r t}{a} .
$$

Let $\left(p_{n}(x)\right)_{n \geq 0}$ be the polynomial sequence associated with $\mathscr{A}=\left(1 /\left(1+r t^{2}\right)\right.$, at $\left./\left(1+r t^{2}\right)\right)$. Then $\left(p_{n}(x)\right)_{n \geq 0}$ satisfies the recurrence relation:

$$
p_{n}(x)=\operatorname{axp}_{n-1}(x)-r p_{n-2}(x), \quad n \geq 2,
$$

with initial condition $p_{0}(x)=1$, and $p_{1}(x)=a x$. In general, $p_{n}(x)$ is also given by the following Hessenberg determinant:

$$
p_{n}(x)=(-1)^{n}\left|\begin{array}{cccccccc}
-a x & 1 & 0 & 0 & 0 & 0 & \cdots & 0 \\
r & -a x & 1 & 0 & 0 & 0 & \cdots & 0 \\
0 & r & -a x & 1 & 0 & 0 & \cdots & 0 \\
0 & 0 & r & -a x & 1 & 0 & \cdots & 0 \\
0 & 0 & 0 & r & -a x & 1 & \cdots & 0 \\
\vdots & \vdots & \vdots & \vdots & \vdots & \vdots & \ddots & \vdots \\
0 & 0 & 0 & 0 & 0 & 0 & \cdots & 1 \\
0 & 0 & 0 & 0 & 0 & 0 & \cdots & -a x
\end{array}\right| .
$$

If $a=1, r=-1$, then $p_{n}(x)$ becomes the Fibonacci polynomials:

$$
F_{n}(x)=(-1)^{n}\left|\begin{array}{cccccccc}
-x & 1 & 0 & 0 & 0 & 0 & \cdots & 0 \\
-1 & -x & 1 & 0 & 0 & 0 & \cdots & 0 \\
0 & -1 & -x & 1 & 0 & 0 & \cdots & 0 \\
0 & 0 & -1 & -x & 1 & 0 & \cdots & 0 \\
0 & 0 & 0 & -1 & -x & 1 & \cdots & 0 \\
\vdots & \vdots & \vdots & \vdots & \vdots & \vdots & \ddots & \vdots \\
0 & 0 & 0 & 0 & 0 & 0 & \cdots & 1 \\
0 & 0 & 0 & 0 & 0 & 0 & \cdots & -x
\end{array}\right| .
$$

If $a=2, r=-1$, then $p_{n}(x)$ gives the Pell polynomials:

$$
\begin{aligned}
P_{n}(x)=F_{n}(2 x) & =(-1)^{n}\left|\begin{array}{cccccccc}
-2 x & 1 & 0 & 0 & 0 & 0 & \cdots & 0 \\
-1 & -2 x & 1 & 0 & 0 & 0 & \cdots & 0 \\
0 & -1 & -2 x & 1 & 0 & 0 & \cdots & 0 \\
0 & 0 & -1 & -2 x & 1 & 0 & \cdots & 0 \\
0 & 0 & 0 & -1 & -2 x & 1 & \cdots & 0 \\
\vdots & \vdots & \vdots & \vdots & \vdots & \vdots & \ddots & \vdots \\
0 & 0 & 0 & 0 & 0 & 0 & \cdots & 1 \\
0 & 0 & 0 & 0 & 0 & 0 & \cdots & -2 x
\end{array}\right| .
\end{aligned}
$$

In case $a=2, r=1, p_{n}(x)$ becomes the Chebyshev polynomials of the second kind:

$$
U_{n}(x)=(-1)^{n}\left|\begin{array}{cccccccc}
-2 x & 1 & 0 & 0 & 0 & 0 & \cdots & 0 \\
1 & -2 x & 1 & 0 & 0 & 0 & \cdots & 0 \\
0 & 1 & -2 x & 1 & 0 & 0 & \cdots & 0 \\
0 & 0 & 1 & -2 x & 1 & 0 & \cdots & 0 \\
0 & 0 & 0 & 1 & -2 x & 1 & \cdots & 0 \\
\vdots & \vdots & \vdots & \vdots & \vdots & \vdots & \ddots & \vdots \\
0 & 0 & 0 & 0 & 0 & 0 & \cdots & 1 \\
0 & 0 & 0 & 0 & 0 & 0 & \cdots & -2 x
\end{array}\right| .
$$

Example 7. Considering the Riordan array $\mathscr{B}=\left(\left(1-b t^{2}\right) /(1+\right.$ $\left.r t^{2}\right)$, at $\left./\left(1+r t^{2}\right)\right)$, we have $\left(\left(1-b t^{2}\right) /\left(1+r t^{2}\right)\right.$, at $/(1+$ $\left.\left.r t^{2}\right)\right)(1 /(1-x t))=\left(1-b t^{2}\right) /\left(1-a x t+r t^{2}\right)$. Then the generating functions of the $A$ - and $Z$-sequences of $\mathscr{B}^{-1}$ are

$$
A(t)=\frac{1+r t^{2}}{a}, \quad Z(t)=\frac{(b+r) t}{a}
$$

Let $\left(p_{n}(x)\right)_{n \geq 0}$ be the polynomial sequence associated with $\mathscr{B}=\left(\left(1-b t^{2}\right) /\left(1+r t^{2}\right)\right.$, at $\left./\left(1+r t^{2}\right)\right)$. Then $\left(p_{n}(x)\right)_{n \geq 0}$ satisfies the recurrence relation:

$$
p_{n}(x)=\operatorname{axp}_{n-1}(x)-r p_{n-2}(x), \quad n \geq 3,
$$

with initial condition $p_{0}(x)=1$, and $p_{1}(x)=a x, p_{2}(x)=$ $a^{2} x^{2}-b-r$.

In general, $p_{n}(x)$ is also given by the following Hessenberg determinant:

$$
p_{n}(x)=(-1)^{n}\left|\begin{array}{cccccccc}
-a x & 1 & 0 & 0 & 0 & 0 & \cdots & 0 \\
b+r & -a x & 1 & 0 & 0 & 0 & \cdots & 0 \\
0 & r & -a x & 1 & 0 & 0 & \cdots & 0 \\
0 & 0 & r & -a x & 1 & 0 & \cdots & 0 \\
0 & 0 & 0 & r & -a x & 1 & \cdots & 0 \\
\vdots & \vdots & \vdots & \vdots & \vdots & \vdots & \ddots & \vdots \\
0 & 0 & 0 & 0 & 0 & 0 & \cdots & 1 \\
0 & 0 & 0 & 0 & 0 & 0 & \cdots & -a x
\end{array}\right| .
$$

If $a=2, b=r=1$, then $p_{n}(x)$ become the Chebyshev polynomials of the first kind $2 T_{n}(x)-0^{n}$ :

$$
2 T_{n}(x)=(-1)^{n}\left|\begin{array}{cccccccc}
-2 x & 1 & 0 & 0 & 0 & 0 & \cdots & 0 \\
2 & -2 x & 1 & 0 & 0 & 0 & \cdots & 0 \\
0 & 1 & -2 x & 1 & 0 & 0 & \cdots & 0 \\
0 & 0 & 1 & -2 x & 1 & 0 & \cdots & 0 \\
0 & 0 & 0 & 1 & -2 x & 1 & \cdots & 0 \\
\vdots & \vdots & \vdots & \vdots & \vdots & \vdots & \ddots & \vdots \\
0 & 0 & 0 & 0 & 0 & 0 & \cdots & 1 \\
0 & 0 & 0 & 0 & 0 & 0 & \cdots & -2 x
\end{array}\right| .
$$


In case $a=3, b=0, r=2, p_{n}(x)$ give the Fermat polynomials (see [15]):

$$
f_{n}(x)=(-1)^{n}\left|\begin{array}{cccccccc}
-3 x & 1 & 0 & 0 & 0 & 0 & \cdots & 0 \\
2 & -3 x & 1 & 0 & 0 & 0 & \cdots & 0 \\
0 & 2 & -3 x & 1 & 0 & 0 & \cdots & 0 \\
0 & 0 & 2 & -3 x & 1 & 0 & \cdots & 0 \\
0 & 0 & 0 & 2 & -3 x & 1 & \cdots & 0 \\
\vdots & \vdots & \vdots & \vdots & \vdots & \vdots & \ddots & \vdots \\
0 & 0 & 0 & 0 & 0 & 0 & \cdots & 1 \\
0 & 0 & 0 & 0 & 0 & 0 & \cdots & -3 x
\end{array}\right| .
$$

Example 8. Considering the Riordan array $\mathscr{C}=((1-b t) /(1+$ $\left.r t^{2}\right)$, at $\left./\left(1+r t^{2}\right)\right)$, we have $\left((1-b t) /\left(1+r t^{2}\right)\right.$, at $\left./\left(1+r t^{2}\right)\right)(1 /(1-$ $x t))=(1-b t) /\left(1-a x t+r t^{2}\right)$. The generating functions of the $A$ - and $Z$-sequences of $\mathscr{C}^{-1}$ are

$$
A(t)=\frac{1+r t^{2}}{a}, \quad Z(t)=\frac{b+r t}{a} .
$$

Let $\left(p_{n}(x)\right)_{n \geq 0}$ be the polynomial sequence associated with $\mathscr{C}=\left((1-b t) /\left(1+r t^{2}\right), a t /\left(1+r t^{2}\right)\right)$. Then $\left(p_{n}(x)\right)_{n \geq 0}$ satisfies the recurrence relation:

$$
p_{n}(x)=\operatorname{axp}_{n-1}(x)-r p_{n-2}(x), \quad n \geq 3,
$$

with initial condition $p_{0}(x)=1$, and $p_{1}(x)=a x-b, p_{2}(x)=$ $a^{2} x^{2}+a b x-r$.

In general, $p_{n}(x)$ is also given by the following Hessenberg determinant:

$$
p_{n}(x)=(-1)^{n}\left|\begin{array}{cccccccc}
b-a x & 1 & 0 & 0 & 0 & 0 & \cdots & 0 \\
r & -a x & 1 & 0 & 0 & 0 & \cdots & 0 \\
0 & r & -a x & 1 & 0 & 0 & \cdots & 0 \\
0 & 0 & r & -a x & 1 & 0 & \cdots & 0 \\
0 & 0 & 0 & r & -a x & 1 & \cdots & 0 \\
\vdots & \vdots & \vdots & \vdots & \vdots & \vdots & \ddots & \vdots \\
0 & 0 & 0 & 0 & 0 & 0 & \cdots & 1 \\
0 & 0 & 0 & 0 & 0 & 0 & \cdots & -a x
\end{array}\right| .
$$

If $a=2, b=1, r=1$, then $p_{n}(x)$ becomes the Chebyshev polynomials of the third kind $V_{n}(x)$ :

$$
V_{n}(x)=(-1)^{n}\left|\begin{array}{cccccccc}
1-2 x & 1 & 0 & 0 & 0 & 0 & \cdots & 0 \\
1 & -2 x & 1 & 0 & 0 & 0 & \cdots & 0 \\
0 & 1 & -2 x & 1 & 0 & 0 & \cdots & 0 \\
0 & 0 & 1 & -2 x & 1 & 0 & \cdots & 0 \\
0 & 0 & 0 & 1 & -2 x & 1 & \cdots & 0 \\
\vdots & \vdots & \vdots & \vdots & \vdots & \vdots & \ddots & \vdots \\
0 & 0 & 0 & 0 & 0 & 0 & \cdots & 1 \\
0 & 0 & 0 & 0 & 0 & 0 & \cdots & -2 x
\end{array}\right| .
$$

If $a=2, b=-1, r=1$, then $p_{n}(x)$ gives the Chebyshev polynomials of the fourth kind $W_{n}(x)$ :

$$
W_{n}(x)=(-1)^{n}\left|\begin{array}{cccccccc}
-1-2 x & 1 & 0 & 0 & 0 & 0 & \cdots & 0 \\
1 & -2 x & 1 & 0 & 0 & 0 & \cdots & 0 \\
0 & 1 & -2 x & 1 & 0 & 0 & \cdots & 0 \\
0 & 0 & 1 & -2 x & 1 & 0 & \cdots & 0 \\
0 & 0 & 0 & 1 & -2 x & 1 & \cdots & 0 \\
\vdots & \vdots & \vdots & \vdots & \vdots & \vdots & \ddots & \vdots \\
0 & 0 & 0 & 0 & 0 & 0 & \cdots & 1 \\
0 & 0 & 0 & 0 & 0 & 0 & \cdots & -2 x
\end{array}\right| .
$$

Finally, considering the Riordan array $\mathscr{D}=((1-b t-$ $\left.c t^{2}\right) /\left(1+t^{2}\right)$, at $\left./\left(1+t^{2}\right)\right)$, we have $\left(\left(1-b t-c t^{2}\right) /\left(1+t^{2}\right)\right.$, at $/(1+$ $\left.\left.t^{2}\right)\right)(1 /(1-x t))=\left(1-b t-c t^{2}\right) /\left(1-a x t+t^{2}\right)$. Then the generating functions of the $A$ - and $Z$-sequences of $\mathscr{D}^{-1}$ are

$$
A(t)=\frac{1+t^{2}}{a}, \quad Z(t)=\frac{b+(c+1) t}{a} .
$$

Let $\left(p_{n}(x)\right)_{n \geq 0}$ be the polynomial sequence associated with $\mathscr{D}=\left(\left(1-b t-c t^{2}\right) /\left(1+t^{2}\right)\right.$, at $\left./\left(1+t^{2}\right)\right)$. Then $\left(p_{n}(x)\right)_{n \geq 0}$ satisfies the recurrence relation:

$$
p_{n}(x)=\operatorname{axp}_{n-1}(x)-p_{n-2}(x), \quad n \geq 3,
$$

with initial condition $p_{0}(x)=1$, and $p_{1}(x)=a x-b$, $p_{2}(x)=a x p_{1}(x)-(c+1) p_{0}(x)=a^{2} x^{2}-a b x-c-1$. For $n \geq 1$, we have

$$
p_{n}(x)=(-1)^{n}\left|\begin{array}{cccccccc}
b-a x & 1 & 0 & 0 & 0 & 0 & \cdots & 0 \\
c+1 & -a x & 1 & 0 & 0 & 0 & \cdots & 0 \\
0 & 1 & -a x & 1 & 0 & 0 & \cdots & 0 \\
0 & 0 & 1 & -a x & 1 & 0 & \cdots & 0 \\
0 & 0 & 0 & 1 & -a x & 1 & \cdots & 0 \\
\vdots & \vdots & \vdots & \vdots & \vdots & \vdots & \ddots & \vdots \\
0 & 0 & 0 & 0 & 0 & 0 & \cdots & 1 \\
0 & 0 & 0 & 0 & 0 & 0 & \cdots & -a x
\end{array}\right| .
$$

Therefore we can give, now, the following.

Definition 9. The Chebyshev polynomial of degree $n$, denoted by $C_{n}(x, a, b, c)$, is defined by

$$
C_{0}(x, a, b, c)=1,
$$

$$
C_{n}(x, a, b, c)
$$

$$
=(-1)^{n}\left|\begin{array}{cccccccc}
b-a x & 1 & 0 & 0 & 0 & 0 & \cdots & 0 \\
c+1 & -a x & 1 & 0 & 0 & 0 & \cdots & 0 \\
0 & 1 & -a x & 1 & 0 & 0 & \cdots & 0 \\
0 & 0 & 1 & -a x & 1 & 0 & \cdots & 0 \\
0 & 0 & 0 & 1 & -a x & 1 & \cdots & 0 \\
\vdots & \vdots & \vdots & \vdots & \vdots & \vdots & \ddots & \vdots \\
0 & 0 & 0 & 0 & 0 & 0 & \cdots & 1 \\
0 & 0 & 0 & 0 & 0 & 0 & \cdots & -a x
\end{array}\right|,
$$

$n \geq 1$, 
where $C_{n}(x, a, b, c)$ is represented by a Hessenberg determinant of order $n$.

Note that $C_{n}(x, 2,0,1)=2 T_{n}(x), C_{n}(x, 2,0,0)=U_{n}(x)$, $C_{n}(x, 2,1,0)=V_{n}(x)$, and $C_{n}(x, 2,-1,0)=W_{n}(x)$. Hence, Definition 9 can be considered as a unified form for the four kinds of Chebyshev polynomials.

\section{Acknowledgments}

The authors wish to thank the editor and referee for their helpful comments and suggestions. This work was supported by the National Natural Science Foundation of China (Grant no. 11261032) and the Natural Science Foundation of Gansu Province (Grant no. 1010RJZA049).

\section{References}

[1] G.-S. Cheon, H. Kim, and L. W. Shapiro, "Combinatorics of Riordan arrays with identical $A$ and $Z$ sequences," Discrete Mathematics, vol. 312, no. 12-13, pp. 2040-2049, 2012.

[2] T. X. He, "Parametric Catalan numbers and Catalan triangles," Linear Algebra and its Applications, vol. 438, no. 3, pp. 14671484, 2013.

[3] T. X. He, L. C. Hsu, and P. J. S. Shiue, "The Sheffer group and the Riordan group," Discrete Applied Mathematics, vol. 155, no. 15, pp. 1895-1909, 2007.

[4] L. W. Shapiro, S. Getu, W. J. Woan, and L. C. Woodson, "The Riordan group," Discrete Applied Mathematics, vol. 34, no. 1-3, pp. 229-239, 1991.

[5] L. W. Shapiro, "Bijections and the Riordan group," Theoretical Computer Science, vol. 307, no. 2, pp. 403-413, 2003.

[6] R. Sprugnoli, "Riordan arrays and the Abel-Gould identity," Discrete Mathematics, vol. 142, no. 1-3, pp. 213-233, 1995.

[7] R. Sprugnoli, "Riordan arrays and combinatorial sums," Discrete Mathematics, vol. 132, no. 1-3, pp. 267-290, 1994.

[8] T. X. He and R. Sprugnoli, "Sequence characterization of Riordan arrays," Discrete Mathematics, vol. 309, no. 12, pp. 39623974, 2009.

[9] D. Merlini, D. G. Rogers, R. Sprugnoli, and M. C. Verri, "On some alternative characterizations of Riordan arrays," Canadian Journal of Mathematics, vol. 49, no. 2, pp. 301-320, 1997.

[10] E. Munarini, "Riordan matrices and sums of harmonic numbers," Applicable Analysis and Discrete Mathematics, vol. 5, no. 2, pp. 176-200, 2011.

[11] P. Peart and W. J. Woan, "Generating functions via Hankel and Stieltjes matrices," Journal of Integer Sequences, vol. 3, article 00.2.1, no. 2, 2000.

[12] W. Wang and T. Wang, "Generalized Riordan arrays," Discrete Mathematics, vol. 308, no. 24, pp. 6466-6500, 2008.

[13] E. Deutsch, L. Ferrari, and S. Rinaldi, "Production matrices and Riordan arrays," Annals of Combinatorics, vol. 13, no. 1, pp. 6585, 2009.

[14] E. Deutsch, L. Ferrari, and S. Rinaldi, "Production matrices," Advances in Applied Mathematics, vol. 34, no. 1, pp. 101-122, 2005.

[15] A. Luzón and M. A. Morón, "Recurrence relations for polynomial sequences via Riordan matrices," Linear Algebra and its Applications, vol. 433, no. 7, pp. 1422-1446, 2010.

[16] F. A. Costabile and E. Longo, "A determinantal approach to Appell polynomials," Journal of Computational and Applied Mathematics, vol. 234, no. 5, pp. 1528-1542, 2010.
[17] Y. Yang, "Determinant representations of Appell polynomial sequences," Operators and Matrices, vol. 2, no. 4, pp. 517-524, 2008.

[18] S. Roman, The Umbral Calculus, vol. 111 of Pure and Applied Mathematics, Academic Press, New York, NY, USA, 1984.

[19] S. 1. Yang, "Recurrence relations for the Sheffer sequences," Linear Algebra and its Applications, vol. 437, no. 12, pp. 29862996, 2012.

[20] K. Aghigh, M. Masjed-Jamei, and M. Dehghan, "A survey on third and fourth kind of Chebyshev polynomials and their applications," Applied Mathematics and Computation, vol. 199, no. 1, pp. 2-12, 2008.

[21] L. Comtet, Advanced Combinatorics, D. Reidel Publishing, Dordrecht, The Netherlands, 1974. 


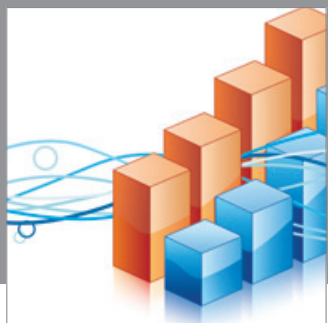

Advances in

Operations Research

mansans

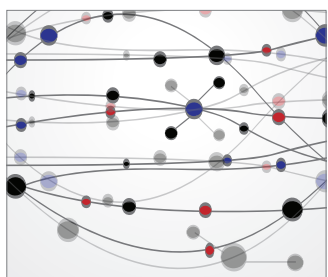

The Scientific World Journal
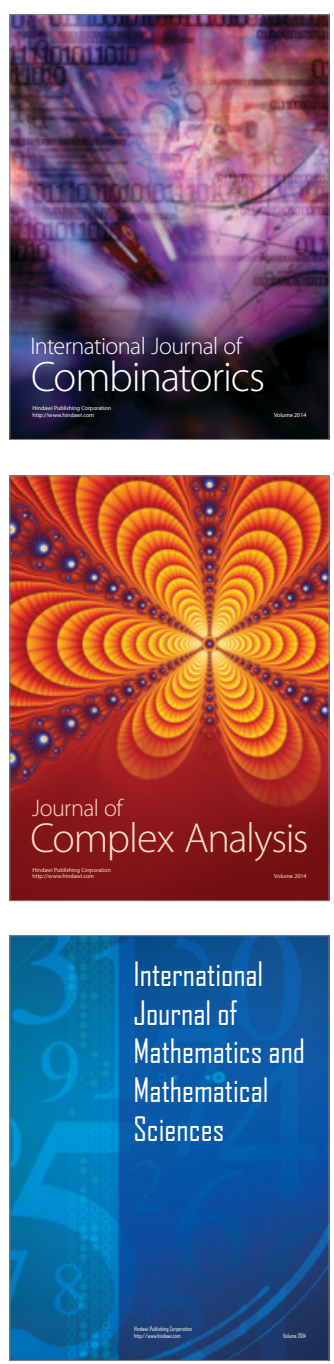
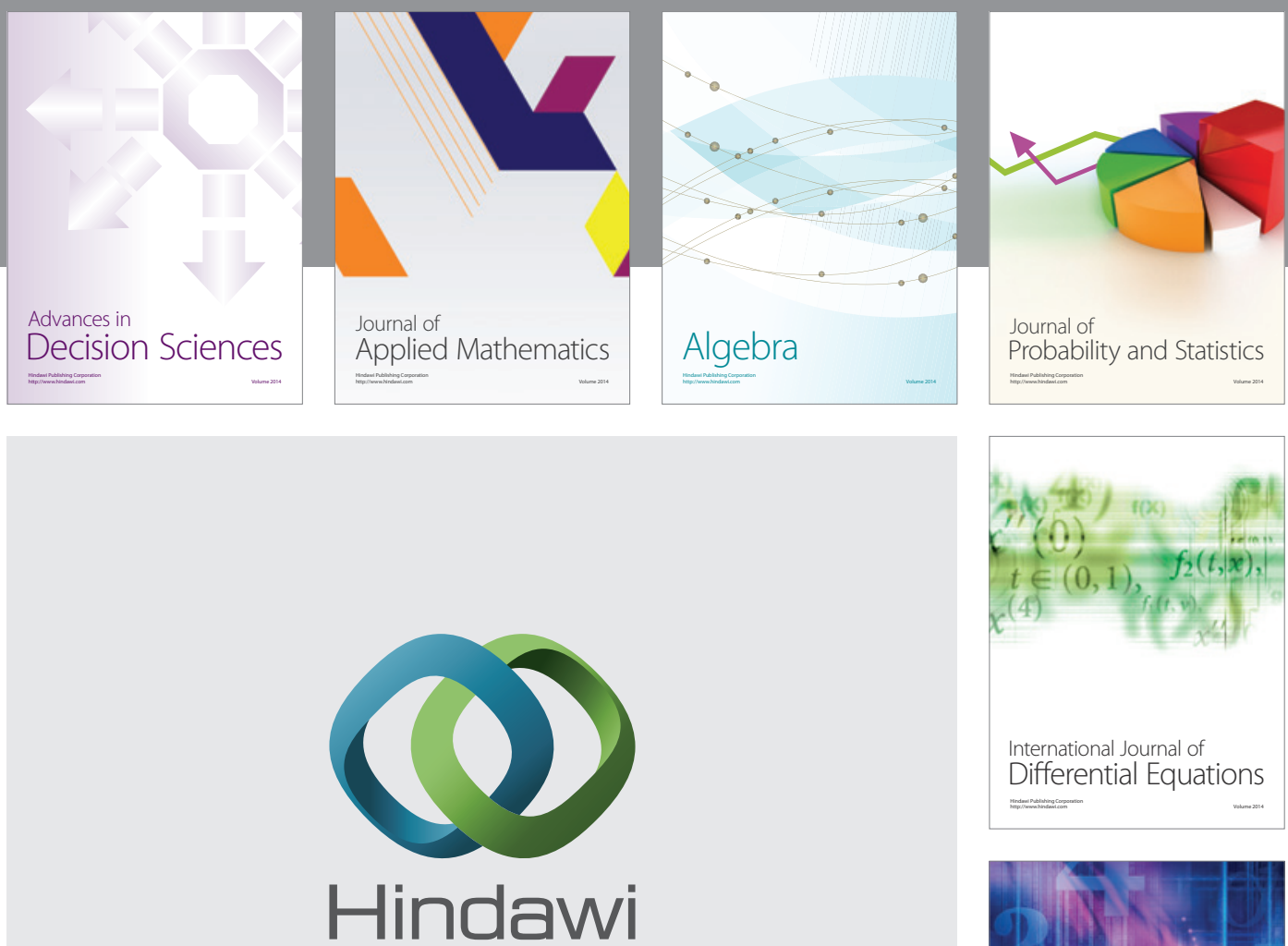

Submit your manuscripts at http://www.hindawi.com
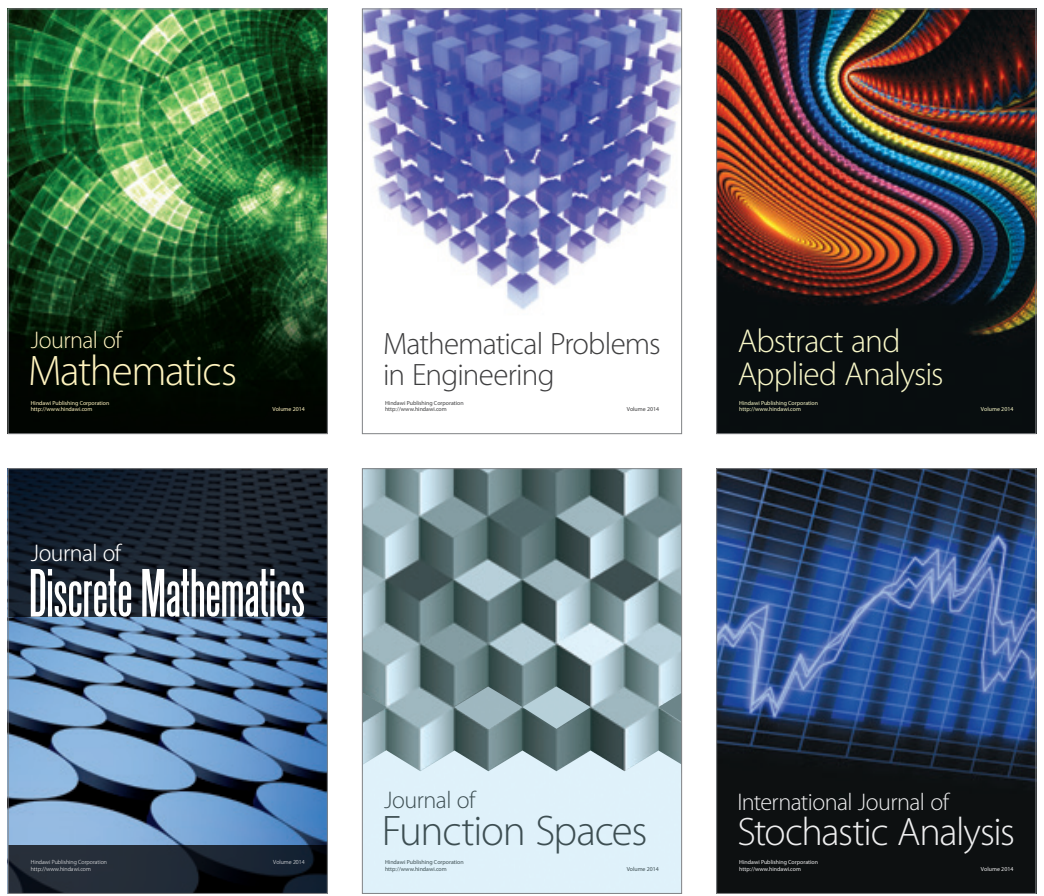

Journal of

Function Spaces

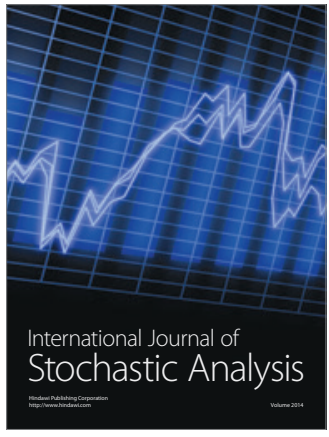

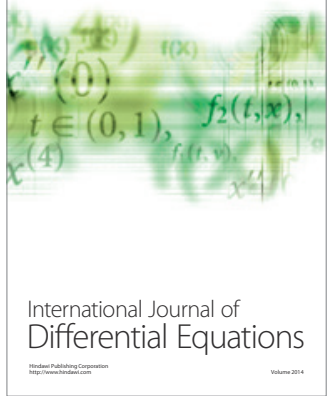
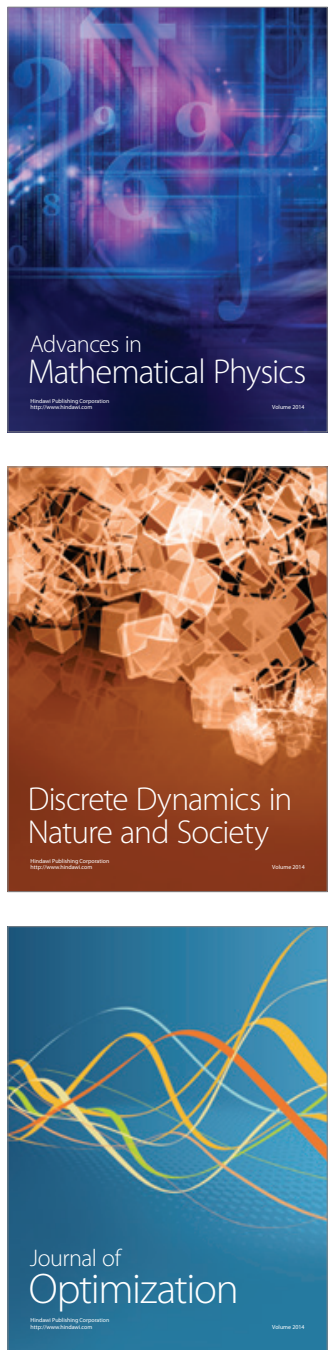